\title{
Sistemas derivados de esquemas nodais em problemas bidimensionais de transporte de partículas: uma análise espectral
}

\author{
Camila B. Picoloto, \\ Programa de Pós-Graduação em Engenharia Mecânica, UFRGS, \\ 90050-170, Porto Alegre, RS \\ E-mail: cbpicoloto@gmail.com \\ Patricia Rodrigues, \\ Departamento de Ciências Agronômicas e Ambientais, UFSM, \\ 98400-000, Frederico Westphalen, RS \\ E-mail: patricia@ufsm.br \\ Rudnei D. da Cunha, Liliane B. Barichello \\ Instituto de Matemática, UFRGS, \\ 91509-900, Porto Alegre, RS \\ E-mail: rcunha@mat.ufrgs.br, lbaric@mat.ufrgs.br.
}

\begin{abstract}
Resumo: Neste trabalho, derivou-se uma abordagem alternativa ao problema de autovalores associado a solução de um problema bidimensional de transporte de partículas em geometria cartesiana, definido em um domínio retangular, contendo fonte de nêutrons isotrópica. A abordagem baseia-se no uso de esquemas nodais juntamente com a aplicação do Método de Ordenadas Discretas Analítico (ADO). O esquema nodal é definido em todo o domínio do problema e o método ADO é aplicado para desenvolver soluções para as equações integradas. Tal metodologia combinada com o ordenamento das direções das partículas, reduz à metade a ordem dos sistemas de autovalores associados, quando comparada a outras abordagens existentes na literatura. Aqui, a estrutura da matriz, no caso de problemas isotópicos, é utilizada para reescrever o problema de forma ainda mais simplificada, como perturbação de uma matriz diagonal, que por suas características inerentes, possibilita ainda maior ganho e eficiência computacional.
\end{abstract}

Palavras-chave: Análise espectral, Método de ordenadas discretas analítico, Transporte de partículas

\section{Introdução}

O método de ordenadas discretas analítico (ADO), proposto por Barichello e Siewert [5], tem sido utilizado com sucesso para resolver de forma concisa e precisa problemas de transporte $[2,3,4,10,13,14,15]$. No caso de problemas muldimensionais, associado com esquemas nodais $[1,6,11]$, não requer o uso de esquemas iterativos e nem a divisão do domínio em células, devido a característica analítica da abordagem utilizada. Além disso, o cálculo de autovalores ocorre a partir de sistemas de ordem reduzida e a solução pode ser escrita de forma explícita na variável espacial. Assim, em termos de comparação com outras abordagens que também utilizam o método de ordenadas discretas, o fato de se ter um problema de autovalores de ordem reduzida, contribui para uma formulação mais eficiente do ponto de vista computacional.

Neste trabalho, diferentemente das abordagens desenvolvidas anteriormente, realiza-se a análise espectral das matrizes associadas ao problema de autovalores, obtidas a partir da solução 
de problemas bidimensionais de transporte de nêutrons, rederivando o problema na forma de uma matriz perturbação de matriz diagonal.

\section{Solução em ordenadas discretas analítica na variável $x$}

Considera-se um problema bidimensional de transporte de nêutrons, homogêneo, em geometria cartesiana bidimensional, definido em uma região retangular $R,[0, a] \times[0, b]$, com espalhamento isotrópico, um grupo de energia e com presença de uma fonte isotrópica $Q(x, y)$ definida no retângulo $[0, a s] \times[0, b s]$ dentro de $R$.

Neste contexto, escreve-se a equação bidimensional de transporte em ordenadas discretas para o caso isotrópico [9]

$$
\mu_{i} \frac{\partial}{\partial x} \Psi\left(x, y, \boldsymbol{\Omega}_{i}\right)+\eta_{i} \frac{\partial}{\partial y} \Psi\left(x, y, \boldsymbol{\Omega}_{i}\right)+\sigma_{t} \Psi\left(x, y, \boldsymbol{\Omega}_{i}\right)=Q(x, y)+\frac{\sigma_{s}}{4} \sum_{k=1}^{M} \omega_{k} \Psi\left(x, y, \boldsymbol{\Omega}_{k}\right)
$$

para $i=1, \ldots, M$, com $M=N(N+2) / 2$, de acordo com o esquema de quadratura simétrica de nível [9], onde $N$ refere-se a ordem da quadratura e $\omega_{i}$ são os pesos associados às direções $\boldsymbol{\Omega}_{i}=\left(\mu_{i}, \eta_{i}\right)$. Além disso, $x$ e $y$ são as componentes do vetor espacial, $Q(x, y)$ é o termo fonte de nêutrons, $\sigma_{t}$ e $\sigma_{s}$ são, respectivamente, seção de choque macroscópica total e de espalhamento.

Objetivando analisar a equação para a direção $x$, optou-se por ordenar o conjunto de direções $\left(\omega_{i}, \boldsymbol{\Omega}_{i}\right)$ de forma que para os índices $i=1, \ldots, M / 2$ as direções tenham a coordenada $\mu_{i}>0$, conforme está esboçado na Figura 1.

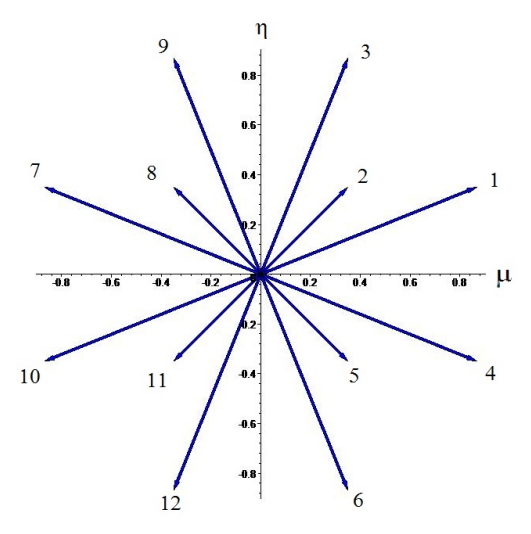

Figura 1: Escolha da numeração das direções para $N=4$

Considerando-se a ordenação das direções apresentada na Figura 1, e utilizando os fundamentos dos métodos nodais para obter as equações unidimensionais na variável $x$, integra-se a equação (1) em todo $y$ sendo escrita na forma

$$
\begin{aligned}
\mu_{i} \frac{d}{d x} \Psi_{y}\left(x, \boldsymbol{\Omega}_{i}\right)+\frac{\eta_{i}}{b}\left[\Psi\left(x, b, \boldsymbol{\Omega}_{i}\right)-\Psi\left(x, 0, \boldsymbol{\Omega}_{i}\right)\right]+\sigma_{t} \Psi_{y}\left(x, \boldsymbol{\Omega}_{i}\right)= \\
Q_{y}(x)+\frac{\sigma_{s}}{4} \sum_{k=1}^{M / 2} \omega_{k}\left[\Psi_{y}\left(x, \boldsymbol{\Omega}_{k}\right)+\Psi_{y}\left(x, \boldsymbol{\Omega}_{k+M / 2}\right)\right]
\end{aligned}
$$

e 


$$
\begin{aligned}
-\mu_{i} \frac{d}{d x} \Psi_{y}\left(x, \boldsymbol{\Omega}_{i+M / 2}\right)+\frac{\eta_{i+M / 2}}{b}\left[\Psi\left(x, b, \boldsymbol{\Omega}_{i+M / 2}\right)-\Psi\left(x, 0, \boldsymbol{\Omega}_{i+M / 2}\right)\right]+ \\
\sigma_{t} \Psi_{y}\left(x, \boldsymbol{\Omega}_{i+M / 2}\right)=Q_{y}(x)+\frac{\sigma_{s}}{4} \sum_{k=1}^{M / 2} \omega_{k}\left[\Psi_{y}\left(x, \boldsymbol{\Omega}_{k}\right)+\Psi_{y}\left(x, \boldsymbol{\Omega}_{k+M / 2}\right)\right],
\end{aligned}
$$

com $i=1, \ldots, M / 2$, onde

$$
\Psi_{y}(x, \boldsymbol{\Omega})=\frac{1}{b} \int_{0}^{b} \Psi(x, y, \boldsymbol{\Omega}) d y
$$

e

$$
Q_{y}(x)=\frac{1}{b} \int_{0}^{b} Q(x, y) d y .
$$

Objetivando-se resolver a parte homogênea das equações unidimensionais (2) e (3) através de um sistema de autovalores, buscam-se, conforme [10, 14], soluções na forma

$$
\Psi_{y}^{h}(x, \boldsymbol{\Omega})=\Phi_{y}(\nu, \boldsymbol{\Omega}) e^{-x / \nu} .
$$

Substituindo, então, a equação (6) nas versões homogêneas das equações (2) e (3), obtém-se

$$
-\frac{\mu_{i}}{\nu} \Phi_{y}\left(\nu, \boldsymbol{\Omega}_{i}\right)+\sigma_{t} \Phi_{y}\left(\nu, \boldsymbol{\Omega}_{i}\right)=\frac{\sigma_{s}}{4} \sum_{k=1}^{M / 2} \omega_{k}\left[\Phi_{y}\left(\nu, \boldsymbol{\Omega}_{k}\right)+\Phi_{y}\left(\nu, \boldsymbol{\Omega}_{k+M / 2}\right)\right],
$$

e

$$
\frac{\mu_{i}}{\nu} \Phi_{y}\left(\nu, \boldsymbol{\Omega}_{i+M / 2}\right)+\sigma_{t} \Phi_{y}\left(\nu, \boldsymbol{\Omega}_{i+M / 2}\right)=\frac{\sigma_{s}}{4} \sum_{k=1}^{M / 2} \omega_{k}\left[\Phi_{y}\left(\nu, \boldsymbol{\Omega}_{k}\right)+\Phi_{y}\left(\nu, \boldsymbol{\Omega}_{k+M / 2}\right)\right]
$$

para $i=1, \ldots, M / 2$. As equações (7) e (8) podem ser escritas como

$$
-\frac{1}{\nu} \mathbf{M} \Phi_{y}\left(\nu, \boldsymbol{\Omega}_{i}\right)=(\mathbf{W}-\mathbf{S}) \Phi_{y}\left(\nu, \boldsymbol{\Omega}_{i}\right)+\mathbf{W} \Phi_{y}\left(\nu, \boldsymbol{\Omega}_{i+M / 2}\right)
$$

e

$$
\frac{1}{\nu} \mathbf{M} \Phi_{y}\left(\nu, \boldsymbol{\Omega}_{i+M / 2}\right)=\mathbf{W} \Phi_{y}\left(\nu, \boldsymbol{\Omega}_{i}\right)+(\mathbf{W}-\mathbf{S}) \Phi_{y}\left(\nu, \boldsymbol{\Omega}_{i+M / 2}\right),
$$

para $i=1, \ldots, M / 2$, onde $\mathbf{S}$ é a matriz diagonal cujos elementos da diagonal principal são as constantes $\sigma_{t}$,

$$
\mathbf{M}=\operatorname{diag}\left\{\mu_{1}, \mu_{2}, \ldots, \mu_{M / 2}\right\}
$$

e

$$
\mathbf{W}(i, j)=\frac{\sigma_{s}}{4} \omega_{j}
$$

Adicionando e subtraindo as equações (9) e (10) e considerando

$$
\mathbf{U}_{y}\left(\nu, \boldsymbol{\Omega}_{i}\right)=\Phi_{y}\left(\nu, \boldsymbol{\Omega}_{i}\right)+\Phi_{y}\left(\nu, \boldsymbol{\Omega}_{i+M / 2}\right)
$$

obtém-se

$$
\left(\mathbf{D}-2 \mathbf{S M}^{-\mathbf{1}} \mathbf{W M}^{-\mathbf{1}}\right) \mathbf{M} \mathbf{U}_{y}=\frac{1}{\nu^{2}} \mathbf{M U}_{y}
$$


onde

$$
\mathbf{D}=\operatorname{diag}\left\{\left[\frac{\sigma_{t}}{\mu_{1}}\right]^{2},\left[\frac{\sigma_{t}}{\mu_{2}}\right]^{2}, \ldots,\left[\frac{\sigma_{t}}{\mu_{M / 2}}\right]^{2}\right\} .
$$

Multiplicando a equação (14) por uma matriz diagonal $\mathbf{T}$ representada por

$$
\mathbf{T}=\operatorname{diag}\left\{\omega_{1}^{1 / 2}, \omega_{2}^{1 / 2}, \ldots, \omega_{M / 2}^{1 / 2}\right\}
$$

obtém-se

$$
(\mathbf{D}-2 \boldsymbol{\Xi}) \hat{\mathbf{X}}=\frac{1}{\nu^{2}} \hat{\mathbf{X}}
$$

onde

$$
\Xi=\mathbf{S M}^{-1} \mathbf{T W T}^{-1} \mathbf{M}^{-1}
$$

$\mathrm{e}$

$$
\hat{\mathbf{X}}=\mathbf{T M U}_{y}
$$

Os elementos da matriz $\mathbf{T}$ foram definidos de forma a tornar a matriz $\boldsymbol{\Xi}$ simétrica. Desta forma, o problema de autovalores pode ser escrito na forma

$$
\left(\mathbf{D}-\frac{\sigma_{t} \sigma_{s}}{2} \mathbf{z z}^{T}\right) \hat{\mathbf{X}}=\lambda \hat{\mathbf{X}}
$$

$\operatorname{com} \lambda=1 / \nu^{2} \mathrm{e}$

$$
\mathbf{z}=\left[\begin{array}{llll}
\left(1 / \mu_{1}\right) \omega_{1}^{1 / 2} & \left(1 / \mu_{2}\right) \omega_{2}^{1 / 2} & \ldots & \left(1 / \mu_{M / 2}\right) \omega_{M / 2}^{1 / 2}
\end{array}\right]^{T}
$$

A forma do problema de autovalor definido na equação (20) é obtida quando aplica-se o método "divide and conquer" [7] em matrizes tridiagonais. Encontrados os autovalores, impõese em (7) e (8) a condição de normalização

$$
\sum_{k=1}^{M / 2} \omega_{k}\left[\Phi_{y}\left(\nu, \boldsymbol{\Omega}_{k}\right)+\Phi_{y}\left(\nu, \boldsymbol{\Omega}_{k+M / 2}\right)\right]=1
$$

e assim é possível escrever de forma explícita, observando que a escolha da quadratura deve garantir que o denominador seja diferente de zero,

e

$$
\Phi_{y}\left(\nu, \boldsymbol{\Omega}_{i}\right)=\frac{\sigma_{s} \nu}{4\left(\sigma_{t} \nu-\mu_{i}\right)}
$$

$$
\Phi_{y}\left(\nu, \boldsymbol{\Omega}_{i+M / 2}\right)=\frac{\sigma_{s} \nu}{4\left(\sigma_{t} \nu+\mu_{i}\right)},
$$

com $i=1, \ldots, M / 2$. Escreve-se a solução em ordenadas discretas para a direção $x$ como

$$
\begin{gathered}
\Psi_{y}^{h}\left(x, \boldsymbol{\Omega}_{i}\right)=\sum_{j=1}^{M / 2}\left[A_{j} \Phi\left(\nu_{j}, \boldsymbol{\Omega}_{i}\right) e^{-x / \nu_{j}}+B_{j} \Phi\left(\nu_{j}, \boldsymbol{\Omega}_{i+M / 2}\right) e^{-(a-x) / \nu_{j}}\right] \\
\Psi_{y}^{h}\left(x, \boldsymbol{\Omega}_{i+M / 2}\right)=\sum_{j=1}^{M / 2}\left[A_{j} \Phi\left(\nu_{j}, \boldsymbol{\Omega}_{i+M / 2}\right) e^{-x / \nu_{j}}+B_{j} \Phi\left(\nu_{j}, \boldsymbol{\Omega}_{i}\right) e^{-(a-x) / \nu_{j}}\right]
\end{gathered}
$$

para $i=1, \ldots, M / 2, x \in[0, a]$. As constantes $A_{j}$ e $B_{j}$ são determinadas a partir de um sistema linear, considerando as condições de contorno do problema, e as constantes de separação $\nu_{j}$ são calculadas a partir dos autovalores obtidos da solução de (20). 


\section{Solução em ordenadas discretas analítica na variável $y$}

Da mesma forma, objetivando analisar a equação para a direção $y$, optou-se por ordenar o conjunto de direções $\left(\omega_{i}, \boldsymbol{\Omega}_{i}\right)$ de forma que para os índices $i=1, \ldots, M / 2$ as direções tenham a coordenanada $\eta_{i}>0$, conforme está esboçado na Figura 2.

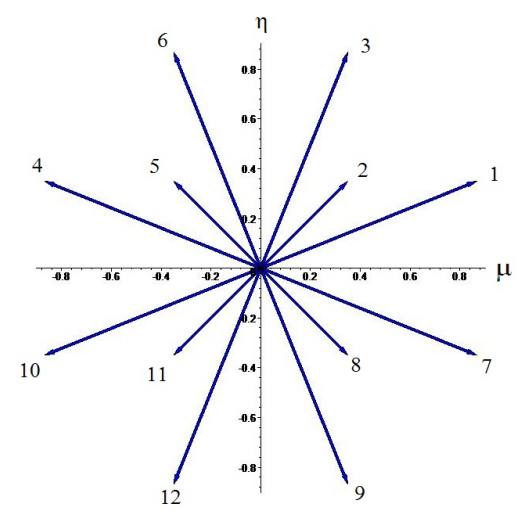

Figura 2: Escolha da numeração das direções para $\mathrm{N}=4$

Considerando-se a ordenação das direções apresentada na Figura 2 e utilizando os fundamentos dos métodos nodais para obter as equações unidimensionais na variável $y$, integrou-se a equação (1) em todo $x$, obtendo-se

$$
\begin{gathered}
\eta_{i} \frac{d}{d y} \Psi_{x}\left(y, \boldsymbol{\Omega}_{i}\right)+\frac{\mu_{i}}{a}\left[\Psi\left(a, y, \boldsymbol{\Omega}_{i}\right)-\Psi\left(0, y, \boldsymbol{\Omega}_{i}\right)\right]+\sigma_{t} \Psi_{x}\left(y, \boldsymbol{\Omega}_{i}\right)= \\
Q_{x}(y)+\frac{\sigma_{s}}{4} \sum_{k=1}^{M / 2} \omega_{k}\left[\Psi_{x}\left(y, \boldsymbol{\Omega}_{k}\right)+\Psi_{x}\left(y, \boldsymbol{\Omega}_{k+M / 2}\right)\right], \\
-\eta_{i} \frac{d}{d y} \Psi_{x}\left(y, \boldsymbol{\Omega}_{i+M / 2}\right)+\frac{\mu_{i+M / 2}}{a}\left[\Psi\left(a, y, \boldsymbol{\Omega}_{i+M / 2}\right)-\Psi\left(0, y, \boldsymbol{\Omega}_{i+M / 2}\right)\right]+ \\
\sigma_{t} \Psi_{x}\left(y, \boldsymbol{\Omega}_{i+M / 2}\right)=Q_{x}(y)+\frac{\sigma_{s}}{4} \sum_{k=1}^{M / 2} \omega_{k}\left[\Psi_{x}\left(y, \boldsymbol{\Omega}_{k}\right)+\Psi_{x}\left(y, \boldsymbol{\Omega}_{k+M / 2}\right)\right],
\end{gathered}
$$

para $i=1, \ldots, M / 2 \mathrm{e}$

$$
\begin{aligned}
\Psi_{x}(y, \boldsymbol{\Omega}) & =\frac{1}{a} \int_{0}^{a} \Psi(x, y, \boldsymbol{\Omega}) d x, \\
Q_{x}(y) & =\frac{1}{a} \int_{0}^{a} Q(x, y) d x .
\end{aligned}
$$

Realizando processo análogo ao descrito na seção anterior, obtém-se o seguinte problema de autovalores

$$
\left(\mathbf{E}-\frac{\sigma_{t} \sigma_{s}}{2} \mathbf{v} \mathbf{v}^{T}\right) \hat{\mathbf{Y}}=\lambda \hat{\mathbf{Y}}
$$

com $\lambda=1 / \nu^{2}$,

$$
\mathbf{E}=\operatorname{diag}\left\{\left[\frac{\sigma_{t}}{\eta_{1}}\right]^{2},\left[\frac{\sigma_{t}}{\eta_{2}}\right]^{2}, \ldots,\left[\frac{\sigma_{t}}{\eta_{M / 2}}\right]^{2}\right\}
$$




$$
\mathbf{v}=\left[\begin{array}{llll}
\left(1 / \eta_{1}\right) \omega_{1}^{1 / 2} & \left(1 / \eta_{2}\right) \omega_{2}^{1 / 2} & \ldots & \left(1 / \eta_{M / 2}\right) \omega_{M / 2}^{1 / 2}
\end{array}\right]^{T}
$$

$\mathrm{e}$

$$
\hat{\mathbf{Y}}=\mathbf{T M U}_{x} .
$$

Impondo-se a condição de normalização

$$
\sum_{k=1}^{M / 2} \omega_{k}\left[\Phi_{x}\left(\nu, \boldsymbol{\Omega}_{k}\right)+\Phi_{x}\left(\nu, \boldsymbol{\Omega}_{k+M / 2}\right)\right]=1
$$

têm-se

$$
\begin{gathered}
\Phi_{x}\left(\nu, \boldsymbol{\Omega}_{i}\right)=\frac{\sigma_{s} \nu}{4\left(\sigma_{t} \nu-\eta_{i}\right)}, \\
\Phi_{x}\left(\nu, \boldsymbol{\Omega}_{i+M / 2}\right)=\frac{\sigma_{s} \nu}{4\left(\sigma_{t} \nu+\eta_{i}\right)},
\end{gathered}
$$

com $i=1, \ldots, M / 2$. A solução em ordenadas discretas para a direção $y$ pode ser escrita como

$$
\begin{gathered}
\Psi_{x}^{h}\left(y, \boldsymbol{\Omega}_{i}\right)=\sum_{j=1}^{M / 2}\left[C_{j} \Phi\left(\nu_{j}, \boldsymbol{\Omega}_{i}\right) e^{-y / \nu_{j}}+D_{j} \Phi\left(\nu_{j}, \boldsymbol{\Omega}_{i+M / 2}\right) e^{-(b-y) / \nu_{j}}\right], \\
\Psi_{x}^{h}\left(y, \boldsymbol{\Omega}_{i+M / 2}\right)=\sum_{j=1}^{M / 2}\left[C_{j} \Phi\left(\nu_{j}, \boldsymbol{\Omega}_{i+M / 2}\right) e^{-y / \nu_{j}}+D_{j} \Phi\left(\nu_{j}, \boldsymbol{\Omega}_{i}\right) e^{-(b-y) / \nu_{j}}\right]
\end{gathered}
$$

para $i=1, \ldots, M / 2, y \in[0, b]$. As constantes de separação $\nu_{j}$ são obtidas a partir dos autovalores obtidos da solução de (31) e as constantes $C_{j}$ e $D_{j}$ são determinadas a partir de um sistema linear, considerando as condições de contorno do problema.

\section{Comentários Finais}

O uso da análise espectral permitiu, além de escrever as soluções elementares do problema de ordenadas discretas de forma explícita, diferentemente de abordagens anteriores, obter um problema de autovalores mais simples. Pela sua natureza, tal sistema pode ser resolvido numericamente de forma mais eficiente $[7,8]$.

Normalmente, problemas de autovalores associados a formulações baseadas no método de ordenadas discretas são da ordem $M$. O emprego do Método ADO possibilita a redução de tais sistemas a ordem $M / 2$. No entanto, a formulação apresentada aqui, reduz o problema de autovalores obtido pelo Método ADO (ordem $M / 2$ ) ao caso mais simples do que de matrizes tridiagonais simétricas, representando relevante ganho em eficiência computacional, o que em problemas multidimensionais é questão significativa.

Resultados obtidos a partir desta abordagem para os problemas de autovalores foram verificados, confirmando os já obtidos na Ref. [12], sendo obtidos com redução relevante de tempo computacional. Objetiva-se, agora, utilizar as soluções elementares para determinação dos fluxos angulares e avaliar quantidades de interesse, como o fluxo escalar, completando a solução do problema bidimensional. 


\section{Referências}

[1] Y. Y. Azmy, The weighted diamond-difference form of nodal transport methods, Nuclear Science Engineering, 98 (1988) 29-40.

[2] L. B. Barichello, Explicit Formulations for Radiative Transfer Problems, em "Thermal Measurements and Inverse Techniques" (H. R. B. Orlande; O. Fudyin; D. Maillet; R. M. Cotta), pp. 541-562, Boca Raton: CRC Press, 2011.

[3] L. B. Barichello; L. C. Cabrera; J. F. Prolo Filho, An analytical approach for a nodal scheme of two-dimensional neutron transport problems, Annals of Nuclear Energy, 38 (2011) 13101317.

[4] L. B. Barichello; M. Camargo; P. Rodrigues; C. E. Siewert, Unified Solutions to Some Classical Flow Problems on the BGK Model, Zeitschrift für Angewandte Mathematik und Physik, 52 (2001) 517-534.

[5] L. B. Barichello; C. E. Siewert, A discrete-ordinates solution for a non-grey model with complete frequency redistribution, Journal of Quantitative Spectroscopy and Radiative Transfer, 62 (1999) 665-675.

[6] R. C. Barros; E. W. Larsen, A spectral nodal method for one-group X,Y-geometry discrete ordinates problems, Nuclear Science and Engineering, 111 (1992) 34-45.

[7] B. N. Datta, "Numerical Linear Algebra and Applications", Brooks/Cole Publishing Co, Pacific Grove, USA, 1995.

[8] G. H. Golub, C. F. V. Loan, "Matrix Computation", The Jonhs Hopkins University Press, Baltimore, 1996.

[9] E. E. Lewis; W. F. Miller, "Computational methods of neutron transport", John Wiley and Sons, New York, 1984.

[10] C. B. Picoloto; A. Tres; R. D. da Cunha; L. B. Barichello, Two-dimensional neutron transport problems with reflective boundary conditions: an analytical approach, "International Nuclear Atlantic Conference", Recife, 2013.

[11] N. Poursalehi; A. Zolfaghari; A. Minuchehr, An adaptive mesh refinement approach for average current nodal expansion method in 2-D rectangular geometry, Annals of Nuclear Energy, 55 (2013) 61-70.

[12] J. F. Prolo Filho; L. B. Barichello, An Analytical Discrete Ordinates Solution for a Nodal Model of a Two-Dimensional Neutron Transport Problem, "International Conference on Mathematics \& Computational Methods Applied to Nuclear Science \& Engineering", pp. 2350-2360, Sun Valley, 2013.

[13] P. Rodrigues; C. H. Kamphorst; L. B. Barichello, A Spectral Method For Rarefied Gas Dynamics Problems in Cylindrical Geometry, International Journal of Pure and Applied Mathematics, 51 (2009) 181-187.

[14] A. Tres; C. Becker; R. D. da Cunha; L. B. Barichello, A deterministic approach for neutron transport in two-dimensional domains, "Congress of Mechanical Engineering", pp. 69226930, Ribeirão Preto, 2013.

[15] A. Tres; C. B, Picoloto; J. F. Prolo Filho, R. D. da Cunha; L. B. Barichello, Explicit formulation of a nodal transport method for discrete ordinates calculations in two-dimensional fixed-source problems, Kerntechnik, 79 (2014) 155-162. 